\title{
Proyectos ambientales escolares: Una alternativa para formar pensamiento crítico desde la educación.
}

Por: Bolívar Cuartas Sandra Milena ${ }^{1}$

Recibido: 19-12-2008

Aceptado: 04-05-2009

El propósito de este ensayo es analizar y discutir las ventajas que otorga la elaboración de un Proyecto Ambiental Escolar, en el cual se propone como tema central, una problemática social, que al lado de la pobreza, la violencia y el desplazamiento forzoso, es quizás una de las que mayor preocupación ha generado y sigue generando en la sociedad colombiana, el consumo de sustancias psicoactivas o drogadicción.

Los Proyectos Ambientales Escolares o PRAES, se han convertido en una alternativa para introducir la dimensión ambiental en el contexto escolar y a la vez han permitido generar espacios para la reflexión y construcción de un pensamiento crítico frente a la realidad. Según Torres (1996) los PRAE son proyectos que desde el aula de clase y desde la institución escolar se vinculan a la solución de la problemática ambiental particular de una localidad o región, permitiendo la generación de espacios comunes de reflexión, desarrollando criterios de solidaridad, tolerancia, búsqueda del consenso, autonomía y, en últimas, preparando para la autogestión en la búsqueda de un mejoramiento de la calidad de vida, que es propósito último de la educación ambiental.

El diseño e implementación de los PRAES, permiten crear y aplicar estrategias de intervención sobre las diversas problemáticas que se generen al interior del contexto educativo. Durante mucho tiempo la idea de una educación ambiental basada en lo naturalista ha permanecido; pero en tiempos como hoy, cuando ya esa concepción ha evolucionado para mostrar perspectivas más contemporáneas, donde la visión ambiental se amplia y trasciende a aspectos como lo económico, lo político, lo cultural, lo social; considerando así todas las dimensiones del ser, se vuelve en una oportunidad para el cambio y la transformación desde la escuela. .

La razón principal por la cual se propone elaborar un PRAE en torno al problema de drogadicción, es porque además de ser una problemática que viene generando gran impacto en instituciones educativas, es un flagelo y una realidad social en la que nuestros jóvenes no están al margen; por lo tanto, es obligación de los centros educativos no continuar exentos de dicha problemática. En este sentido, el PRAE se muestra como alternativa de solución, a través del cual se pueda seguir otorgando la formación integral, que es propósito de toda educación.

${ }^{1}$ Licenciada en educación básica con énfasis en Ciencias Naturales y Educación Ambiental. Universidad de Antioquia. zamiboku@gmail.com 
Esta propuesta toma valor en la medida en que solo unas pocas investigaciones presentan como eje central asuntos que se desligan totalmente de lo ecologista o naturalista, entiéndase aquí naturalismo o ecologismo como una visión de la educación ambiental que se reduce al estudio de los factores que constituyen un ecosistema (biocenosis y biotopo). En tal caso, el desarrollo y aplicación de un PRAE como el que se muestra aquí, toma significado en la medida en que su diseño y ejecución corresponden a una visión holística de la dimensión ambiental y a la cual es inherente lo social.

Este ensayo es entonces el resultado de una experiencia sobre la elaboración de un PRAE con las características que ya se han mencionado, el cual fue implementado en el Colegio Luján de la Ciudad de Medellín, cuyo periodo de tiempo para su diseño y aplicación fue durante el año 2008.

A continuación se hará una síntesis de dicha experiencia, con la cual se espera lograr fortalecer aún más la idea de que la educación ambiental desde una perspectiva contemporánea, logra trastocar el contexto local como parte de un contexto global.

El objetivo principal de la experiencia fue proporcionar a la comunidad educativa elementos conceptuales, actitudinales y valorativos que permitieran a sus miembros asumir una posición critica frente a la problemática a tratar, como es la drogadicción.

Desde el inicio de la investigación se intuye, que dar solución a una problemática tan acentuada como es el consumo de sustancias psicoactivas, es difícil, sobre todo cuando el proyecto es diseñado para ser aplicado en un periodo de tiempo correspondiente a un año escolar; por tal razón nuestra pretensión apuntaba a focalizarse en el ejercicio de formar para la prevención, más que para alcanzar una solución inmediata, ya que siendo conscientes de ello, la solución requeriría de otro tipo de estrategias diferentes a las empleadas y de un mayor tiempo de intervención.

El colegio Luján es una institución educativa no oficial que ofrece diferentes servicios como son cursos de PREICFES, PREUNIERSITARIO, capacitación para el ECAES en diferentes programas, básica secundaria entre otros.

Dicha institución ha prestado sus servicios a la comunidad por un tiempo aproximado de 24 años, lo que le ha permitido configurase en un espacio para la formación de las futuras generaciones de nuestro país.

En consideración con las características de la investigación, esta obedeció a una metodología cualitativa, en particular, se encuentra inscrita en el marco de la investigaciónacción participativa, para la que según De Miguel citado por Rodríguez et al (1999), "se caracteriza por un conjunto de principios, normas y procedimientos metodológicos que permite obtener conocimientos colectivos sobre una determinada realidad social".

Para el trabajo de investigación, se elaboró un diseño metodológico que obedeció a tres etapas:

Para la primera etapa, se diseñó un cuestionario de 12 preguntas orientadas a indagar sobre la concepción que tenia la comunidad educativa, frente al consumo de sustancias psicoactivas; esencialmente lo que se pretendía hallar durante este diagnóstico era si los 
diferentes miembros que constituyen los estamentos académicos veían la drogadicción como un problema relevante en la misma y si consideraban la necesidad de trabajar en torno a esta problemática.

El interés principal con el diagnóstico, era orientar de manera coherente las actividades, talleres y programas que se proponían como estrategia metodológica y que obedecieran a los propósitos y necesidades de la comunidad educativa.

Para la segunda etapa y de acuerdo con el diagnóstico realizado inicialmente, se diseñaron y aplicaron las estrategias metodológicas que permitieron dar cumplimiento a cada uno de los objetivos propuestos.

En primer lugar, se propuso una conferencia-taller donde se tuvo como objetivo enriquecer el conocimiento que docentes, empleados no docentes, administradores y directivas tenían frente al problema del consumo de sustancias psicoactivas, luego para darle continuidad y extender ese conocimiento que ya había sido adquirido, cada docente debió diseñar y aplicar actividades, talleres o conversatorios en cada uno de los grados, mostrando así la relación que existe entre cada una de las áreas de conocimiento con el fenómeno de interés, como es la drogadicción.

En este sentido; se fortaleció el carácter interdisciplinario que se le quiso dar a la propuesta, y con el fin de lograr una comunicación entre los distintos docentes, se planteó que en las reuniones programadas se socializaran las actividades que se venían desarrollando desde cada una de las áreas, e incluso se entregaron informes periódicos que daban cuenta del trabajo que se estaba realizando con los estudiantes.

Por otro lado, para lograr un mayor impacto de la propuesta, se planteó por parte de la coordinadora de disciplina, Laura Santamaría y la docente en el área de Humanidades y Lengua Castellana, Natalia Llano, la posibilidad de realizar un concurso de carácter institucional, en el que se promovieran las habilidades escriturales de los estudiantes a través de la elaboración de un cuento, que debía tener como tema central el problema de las drogas.

El concurso tomó fuerza gracias al apoyo inmediato de las directivas escolares, y con la divulgación e invitación por parte de los agentes involucrados en el proceso, "DROGA TU CUENTO", nombre del concurso, tuvo uno de los mejores impactos. Tanto fue así; que en una de las reuniones programadas, el Doctor José Luján, rector de la institución educativa propuso como estimulación, la publicación de un texto, donde se copilaron los mejores cuentos de los estudiantes y a su vez, para mostrar el interés de los docentes, también se abrió un espacio para las publicaciones poéticas de los mismos, incluyendo los directivos, al final; el texto fue entregado a los padres de familia de cada uno de los estudiantes.

Posteriormente; se planteó también la necesidad de aproximarse a la realidad del otro, pues como bien se sabe, la drogadicción afecta al individuo desde el punto de vista social, dificultando la interacción y la convivencia, es así que surgió la idea de intercambiar los roles, es decir, que los docentes asumieran por un momento el papel de estudiantes y que por el contrario, los estudiantes adoptaran el rol de docentes, para que conocieran las dificultades y valoraran los logros desde uno y otro punto de vista. 
Esta experiencia, fue enriquecedora para los participantes porque permitió conocer los intereses, propósitos, sentimientos y necesidades de los otros, fortaleciéndose así la comunicación entre docentes y estudiantes, aspecto que en últimas tiene relación directa con las actitudes, valores y comportamientos entre los sujetos implicados.

Finalmente se propuso la visita a la sede campestre del Colegio Luján donde se desarrollaron actividades como dinámicas de integración, carreras de observación, concursos y conversatorios relacionados con la convivencia y la drogadicción.

Para la tercera etapa, se presentó un informe general a la comunidad sobre los logros que se habían obtenido durante la realización de las actividades metodológicas planteadas en el PRAE, los medios de divulgación fueron carteleras y reuniones informativas. Por otro lado, se colocó un buzón en el cual la comunidad educativa podía depositar sugerencias que contribuyeran al fortalecimiento del PRAE. Este diagnóstico final se hizo además a través de entrevistas.

También, se produjo un informe a manera de plegable de los resultados obtenidos durante la aplicación y desarrollo del PRAE, los logros y las dificultades presentadas durante el mismo, en tal caso, el plegable fue entregado a cada uno de los agentes implicados en el proceso y el informe de la investigación fue expuesto y divulgado a toda la comunidad educativa.

Respecto a los instrumentos y técnicas utilizados para la recogida de datos, se utilizó el diario de campo, las entrevistas no estructuras y cuestionarios que fueron almacenados para su posterior sistematización y análisis a través de cuadros de información y redes sistémicas.

De acuerdo con el análisis realizado, se obtuvieron los siguientes resultados.

1. El diagnóstico inicial llevado a cabo en la etapa uno, arrojó lo siguiente:

a) Los encuestados en su mayoría (95\%) coincidieron en que el consumo de sustancias psicoactivas es un problema grave que necesita de una intervención directa por parte de la institución, y a su vez plantearon también diversas estrategias de intervención como: formar para la prevención, difundir información sobre el tema que se estaba abordando y brindar acompañamiento constante a través de diferentes mecanismos como conferencias, charlas, actividades, talleres, dinámicas, campañas y conversatorios.

b) Se consideró importante indagar acerca de la percepción que la comunidad educativa tenia frente al consumo de sustancias psicoactivas, por lo que se pudo identificar que la drogadicción era vista como un problema (50\%), una enfermedad (25\%) y/o una adicción (25\%) que afecta al organismo en diferentes aspectos como son: lo físico, psicológico, social, familiar y académico.

c) Los diferentes estamentos institucionales, coincidieron (98\%) en su mayoría con que la realización del PRAE, era pertinente porque permitía abordar una de las problemáticas que más estaba afectando a la institución y que ha sido la principal preocupación de la misma. 
Los resultados obtenidos al finalizar la etapa 1 fueron importantes porque no solo se identificó el problema que más afectaba a la institución, sino que muchos de los encuestados coincidieron en la necesidad de intervenir a través de un proyecto conjunto que permitiera plantear y aplicar estrategias para su posible solución.

La participación, el compromiso y el trabajo en equipo fueron características claves para el desarrollo del proyecto, porque permitió un mayor acercamiento y comunicación entre los diferentes estamentos institucionales que en situaciones anteriores parecían ser ejes independientes que carecían de interrelación. En este sentido, cada estamento que constituye y hace parte de una institución escolar es un pequeño engranaje que funciona en la medida en que cada uno de ellos lo haga también, pero esto solo es posible a través del trabajo conjunto, donde la comunicación juega un papel fundamental y más si la plataforma sobre la que se construye el cambio tiene relación con la investigación en la escuela.

La etapa 2 donde se llevó a cabo cada una de las estrategias metodológicas propuestas arrojó resultados importantes como:

Las conferencias-taller fueron una estrategia clave, porque entre los diferentes estamentos se había llegado a la conclusión de que era necesario llevar a cabo un proceso de capacitación, ya que la mayoría de los estamentos (98\%) reconocieron no estar preparados para afrontar las situaciones que se pudieran presentar en el aula en relación a problemas de consumo de sustancias psicoactivas.

La capacitación continua y permanente es fundamental, si se considera que los estudiantes que están llegando hoy día al aula de clase son un entramado de realidades complejas, donde cada uno representa un universo único con una forma diferente a la del otro de ver y entender el mundo en el cual vive. Por tal razón, el docente esta obligado a emprender una relación de comunicación continua con esas realidades, de otro modo no sería posible.

Cada docente desde el área o disciplina que impartía, realizaba diversas actividades de acuerdo a los conocimientos previamente adquiridos en los talleres de capacitación.

Es importante aclarar que cada actividad diseñada y aplicada era previamente evaluada por un comité de trabajo conformado por los docentes de cada área, evidenciándose así un trabajo interdisciplinario que se extendía al aula de clase, por lo tanto, cada actividad desarrollada por los docentes en el aula era posteriormente socializada en reuniones programadas de profesores donde se discutían los resultados y las experiencias, con el fin de seguir fortaleciendo el proceso formativo que se estaba presentando.

El trabajo interdisciplinario es fundamental para el desarrollo de propuestas en educación ambiental, lo que significa que las plataformas de comunicación que se crean entre una y otra área fortalecen el trabajo colaborativo y le otorgan a la propuesta una perspectiva integral, la cual se convierte en la principal característica de este PRAE. 
a) Se llevó a cabo un intercambio de roles donde los estudiantes asumían el rol de docentes o administradores y los docentes a su vez se colocaban en el papel de estudiantes. Durante esta experiencia los estudiantes expresaron las sensaciones, dificultades y fortalezas de ser profesor y los profesores en su rol de estudiantes manifestaron que "la actividad fue la oportunidad ideal para acercarse más a los estudiantes y fomentar diferentes valores". El principal logro de esta actividad fue el fortalecimiento de los procesos comunicativos entre estudiantes y profesores.

La formación implica además de adquisición de los conocimientos, fomentar y alimentar el ser espiritual que hay en cada uno de nosotros. Con esto me refiero a que los valores y en general, la formación humana es el complemento a ese conocimiento científico que hace que un individuo sea considerado como un sujeto integro en todas sus dimensiones.

b) DROGA TU CUENTO, fue un concurso que se llevó a cabo a nivel institucional donde cada estudiante postulaba un cuento o historieta, previamente asesorado por la docente en el área de Humanidades y Lengua Castellana.

Este concurso fue el que generó quizás mayor impacto sobre la comunidad, ya que la participación de los estudiantes fue absoluta (100\%) e incluso directivos docentes y docentes también se involucraron de forma directa.

En términos muy generales se podría decir que el resultado más destacado en este caso, fue el libro de cuentos y poesías producto del trabajo y la participación inminente de los agentes implicados; que además de haber permitido crear espacios para desarrollar habilidades escriturales, se convirtió también en una fuente de comunicación de lo que pensaban los estudiantes acerca del consumo de sustancias psicoactivas hacia la comunidad educativa en general.

c) También se realizaron actividades como carreras de observación, juegos de roles, cartas, etc., que permitieron un mayor acercamiento interpersonal entre los estudiantes y profesores, con el fin de contribuir a una buena convivencia escolar.

En este caso, se logró dar cumplimiento al objetivo general de proyecto donde la idea inicial era otorgar elementos conceptuales y actitudinales a los estudiantes, con el fin de que a través del pensamiento crítico que se estaban formando estuvieran en la capacidad de tomar decisiones autónomas que según criterios individuales les convenía más. Sin embargo, el proyecto no sólo permitió formar pensamiento crítico, también se fomentaron valores con el fin de otorgarle integridad al trabajo formativo que se venia desarrollando.

Como conclusiones del proyecto encontramos:

El desarrollo de las diferentes estrategias permitió proporcionar elementos conceptuales, actitudinales y valorativos que favorecieron la elaboración de un pensamiento crítico frente a la problemática tratada. 
En tal caso; contribuir a la formación de pensamiento crítico a través de una propuesta como la que aquí se presenta, implica desde el punto de vista educativo formar sujetos autónomos, capaces de tomar decisiones frente a diferentes situaciones de la vida, individuos que reconocen, comprenden y analizan la realidad con criterios que ya se han formado.

- Se logró desarrollar un trabajo interdisciplinar que permitió un acercamiento a la problemática desde diferentes perspectivas, con el fin de permitir una formación integral de cada uno de los estamentos involucrados en el proceso. En este sentido, el trabajo interdisciplinario representa para la investigación y la educación ambiental, la posibilidad de fortalecer el trabajo colaborativo e individual de cada docente.

- La capacitación se convirtió en el eje fundamental para el desarrollo de este proyecto, puesto que a partir de lo aprendido se diseñaron las diferentes estrategias que mejor se adaptaban a las condiciones e intereses de la comunidad educativa y además; permitió que cada estamento involucrado estuviera preparado para afrontar situaciones como las que se vivieron.

- El desarrollo de actividades relacionadas con el encuentro personal y la convivencia permitieron crear espacios de participación, fundamentales para el desarrollo integral del sujeto.

- La capacitación, el trabajo interdisciplinario y la participación constante de los estamentos implicados permitieron alcanzar una mirada crítica frente a la realidad que se vive tanto en el contexto inmediato como en el global, además, favoreció un mayor compromiso y una comprensión de la dimensión ambiental, que se constituyó en una visión sistémica de la realidad.

Esta experiencia descrita anteriormente, sustenta la idea de que los PRAE son en definitiva una alternativa que permite una verdadera intervención a partir de los espacios de reflexión y participación que se generan al interior de los centros educativos y sobre todo muestra, que la educación ambiental permite trascender los limites hacia una realidad más próxima del ser, que le facilita un encuentro de si mismo con aquello que vive a diario.

Los PRAE con enfoque holístico, permiten la formación de pensamiento crítico porque desde esta perspectiva, se considera al sujeto como eje de partida para empezar el cambio que se busca; ya que es él, desde su individualidad y existir el que decide cual es el camino que debe recorrer; en este caso, la escuela como centro formador se debe hacer responsable, en parte, de cada sujeto que educa, pero no de las decisiones que él como sujeto tome para su vida. El papel de la escuela es orientar y otorgar una formación integral al educando, pero es responsabilidad del sujeto mismo concientizarse, con base a lo que ya le fue enseñado, que nadie decidirá por él.

Los seres humanos somos un entramado de realidades complejas, vivimos en un mundo donde lo blanco se mezcla con lo negro, la verdad con la mentira, lo real con lo irreal; es así que cada día de nuestras vidas se torna de tonalidades distintas. Somos organismos vivos capaces de sentir, de existir, son éstas características inherentes a nuestra especie, recorremos un camino cuya ruta desconocemos y sin embargo; para no creer que caminamos a ciegas, trazamos rutas 
de nuestro destino, moldeamos nuestro existir tal como queremos, pero no siempre se toma el camino correcto.

Somos tan humanos, que necesitamos de algo o de alguien para recorrer ese camino. Elegir es difícil, es algo que solo se aprende durante, las decisiones que tienen que ver con cada una de nuestras vidas, son solo así, nuestras. Por tales razones, educar no implica tomar las decisiones por otro, es así que a través de la educación se enseña lo que puede ser correcto o lo que no, lo demás es responsabilidad del sujeto que decide cómo quiere vivir.

Así, el pensamiento crítico que se pueda desarrollar en el sujeto desde la escuela, va a marcar un paso fundamental en su vida, de ahí radica la importancia de proyectos que se focalicen en el sujeto como eje central y que respondan a las necesidades, intereses y dificultades del mismo.

Un individuo capaz de tomar las mejores decisiones para su vida, es con plena seguridad un agente protagonista y transformador de una sociedad que se construye.

En conclusión; podría decirse que los PRAE con enfoque holístico, no solo se caracterizan por ser transdisciplinarios y transversales como ya se mostró a través de la experiencia, son en pocas palabras, una alternativa que permite fomentar pensamiento crítico frente a la realidad que se vive; además facilita el desarrollo de estrategias que buscan humanizar cada vez más los procesos de formación que se llevan a cabo en las instituciones de educación y a su vez, trastocan el contexto local como parte de un contexto global.

\section{Bibliografía}

- RODRIGUEZ G, GIL F, Javier y Eduardo GARCIA J. 1999. Metodología de la investigación cualitativa. Ediciones ALJIBE, Málaga.378 p

- SAUVÉ, L. $2004 . \quad$ Una cartografía de corrientes en educación ambiental. http://www.ambiente.gov.ar/infotecaea/descargas/sauve01.pdf

Fecha última revisión: 31 de octubre de 2008 Fecha último acceso: 4 diciembre 2008

- TORRES, M. 1996. La dimensión ambiental: un reto para la educación de la nueva sociedad : proyecto ambientales escolares una estrategia para la inclusión de la dimensión ambiental en la escuela. Ministerio de Educación Nacional, Bogotá. 98 p 